\title{
Gastric Per-Oral Endoscopic Myotomy (G-POEM) for the Treatment of Gastroparesis: A Single Center Experience
}

\author{
Lennon Gregor ${ }^{1}$, John Wo², John DeWitt ${ }^{2}$, Anita Gupta ${ }^{2}$, Brandon Yim², Martha \\ Mendez ${ }^{2}$, Mohammad Al-Haddad ${ }^{2}$ \\ ${ }^{1}$ Indiana University School of Medicine, ${ }^{2}$ Division of \\ Gastroenterology/Hepatology, Indiana University School of Medicine, \\ Indianapolis, Indiana, USA.
}

\section{Background and Hypothesis:}

Gastroparesis is a difficult-to-treat disease that has variable presentation.

Treatments for gastroparesis include pharmacotherapy, botulinum toxin injection into the pylorus, or surgical pyloroplasty. Based on patient response to pyloric directed therapies, it is theorized that endoscopic pyloromyotomy will prove to be an effective minimally invasive treatment. Early feasibility studies of endoscopic pyloromyotomy (G-POEM) demonstrated safety and early clinical success. Our aim is to assess the safety and sustained clinical efficacy of the G-POEM procedure.

\section{Experimental Design or Project Methods:}

Patients with confirmed diagnosis of gastroparesis on gastric scintigraphy were assessed using PAGI-SYM to determine symptom severity. EndoFLIP of pylorus and four-hour solid phase gastric emptying scan (GES) were utilized to access gastric function pre and post G-POEM. Post G-POEM follow up at 1, 3, 6, and 12 months was completed. PAGI-SYM was collected at all follow ups. For 6- and 12month visits, GES and EndoFLIP were conducted.

\section{Results:}

Twenty-eight patients (age: $28-80$, $82 \%$ female) underwent G-POEM between February 2018 to July 2019. All cases were done under general anesthesia with technical success rates of $100 \%$. There were adverse events in 3/28 of the cases that were successfully managed endoscopically. There was significant improvement in gastric emptying post G-POEM (4-hour average retention rates of $6.3 \%$ compared to $35.0 \%$ pre-GPOEM, $p<0.001)$. There was a significant improvement in patient reported PAGI-SYM score from baseline (3.0) and 3months post G-POEM (0.80), $p<0.001$. There was a trend towards sustained improvement in PAGI-SYM score on 6 month follow up, but this did not reach statistical significance $(2.10, p=0.22)$. There were improvements noted in pylorus cross sectional area and distensibility index on EndoFLIP post G-POEM, but this did not reach statistical significance and will require further assessment and a larger sample size.

\section{Conclusion and Potential Impact:}

G-POEM appears to be a safe and feasible treatment alternative for gastroparesis with significant short-term improvements in gastric emptying rates 
and overall symptom scores. A larger cohort of patients is needed to validate these findings and examine the long-term effect of G-POEM on patients' wellbeing and healthcare utilization.

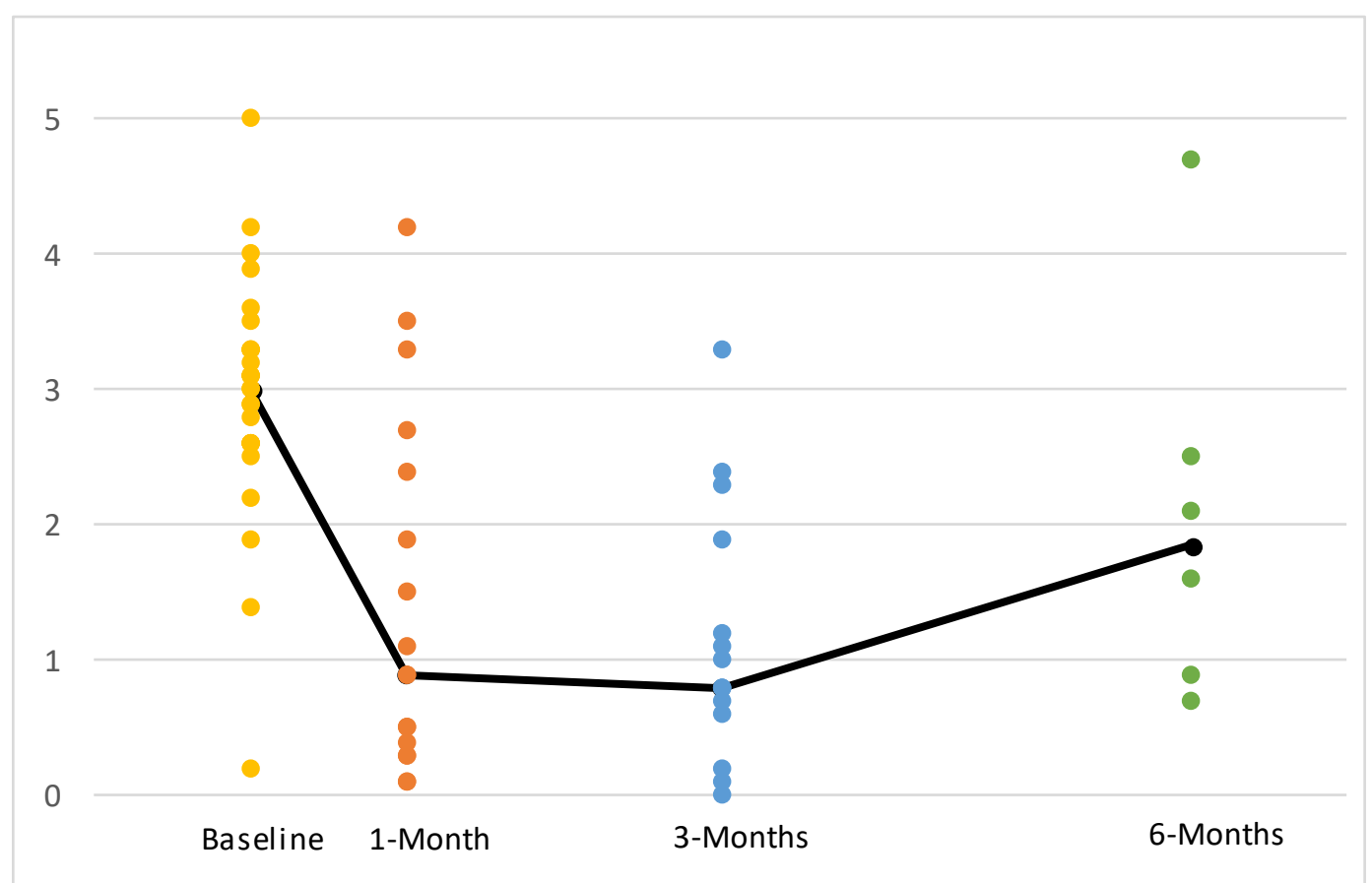

Figure 1. Graph of PAGI-SYM scores at baseline, 1-month, 3-months, and 6months with moving median. 